\title{
The Alterations in Peripheral Blood Components of Women with Abnormal Cervical Cytology
}

\author{
Mine KİSELİ ${ }^{1}$, Aslı YARCI GÜRSOY ${ }^{1}$, Egemen AKINCIOĞLU², Emre Göksan PABUCCU1, Gamze Sinem ÇAĞLAR \\ Hande Esra KOCA ${ }^{1}$, Zehra EDEBAL ${ }^{2}$, Haldun UMUDUM²
}

Ankara, Turkey

\begin{abstract}
OBJECTIVE: To investigate the peripheral blood components, neutrophil/lymphocyte ratio (NLR) and platelet/lymphocyte ratios (PLR) in cervical cytological abnormalities.

STUDY DESIGN: We retrospectively analyzed 185 cases with abnormal and 303 cases with normal cervicovaginal smears (CVS) with blood count profile, from a total number of 9286 patients.

RESULTS: Abnormal CVS results consisted of 137 (72\%) ASC-US, 16 (8\%) ASC-H, 19 (10\%) L-SIL, 4 (2\%) H-SIL and 9 (4\%) AGUS. NLR and PLR of abnormal CVS group were higher than control group $(p<0.001)$. The lymphocyte count was significantly lower in abnormal CVS cases $(p<0.001)$. The number of patients with neutrophilia (neutrophils $\geq 70 \%$ of the total leukocytes) was similar among two groups $(\mathrm{p}>0.05)$.
\end{abstract}

CONCLUSION: Neutrophilia is not observed in cervical preinvasive lesions whereas it is common in cervical cancer. In this study, absence of increased neutrophil counts and presence of lymphopenia in patients with abnormal cervical cytology, may indicate an early measure in cervical pathologies.

Keywords: Cervicovaginal smear, Atypical squamous cells of undetermined significance, Neutrophil/lymphocyte ratio, Platelet/lymphocyte ratio

Gynecol Obstet Reprod Med 2016;22:36-39

\section{Introduction}

Cervical cancer is one of the most frequently occuring cancers in women worldwide, and it is more commonly reported in developing countries. Screening and early detection of the preinvasive disease are crucial for cervical cancer prevention. Cytological abnormalities in cervicovaginal smears (CVS) necessitate follow-up or diagnosis with a colposcopic examination-guided cervical biopsy. Lesions confined to the cervical epithelium are defined as preinvasive disease of the cervix that may be diagnosed and treated prior to the onset of invasive cancer.

Neoplasia pathogenesis has been shown to be related to exacerbated activation and the maintenance of inflammatory

\footnotetext{
1 Ufuk University Faculty of Medicine Department of Obstetrics and Gynecology, Ankara

${ }^{2}$ Ufuk University Faculty of Medicine Department of Pathology, Ankara

Address of Correspondence: Mine Kiseli

Ufuk University Faculty of Medicine

Department of Obstetrics and

Gynecology, Balgat

Ankara, Turkey

minekiseli@gmail.com

Submitted for Publication

25.01.2016

Accepted for Publication:

24.03 .2016
}

pathways. In cancer, local and systemic inflammatory responses have been reported previously. ${ }^{1}$ This nonspecific trait had been observed in various aspects of cancer such as cancer initiation and progression. On the basis of this theory, researchers have studied inflammatory markers as indicators of invasion in different types of cancer. ${ }^{2-5}$ Changes in peripheral blood components such as neutrophils, lymphocytes, platelets and the neutrophil/lymphocyte ratio (NLR) and platelet/lymphocyte ratios (PLR) have been determined to be simple, applicable and reliable prognostic markers of systemic inflammatory response in cancer. ${ }^{6}$ The NLR and PLR have been studied as prognostic and predictive indicators in various types of cancer. ${ }^{6-11}$

In women with gynecological cancer, the NLR, which is measured prior to treatment, has been found to be predictive of survival in patients with ovarian and uterine cervical cancer. ${ }^{12-14}$ Additionally, in cervical cancer patients, the NLR has been shown to predict response to chemotherapy and radiotherapy. ${ }^{15}$ However, little is known about the NLR and PLR in preinvasive diseases of the cervix, and there have been no reports about the predictive value of these inflammatory markers in abnormal cervical cytology. Therefore, in this study we investigated the relationship between inflammatory markers, NLR, PLR, and cervical cytological abnormalities. 


\section{Material and Method}

We retrospectively analyzed the pathological archives of a university hospital with dates between June 2006 and December 2014. Cytological analysis was performed by two pathologists, and we classified cytological abnormalities according to the Bethesda system (2001). From a total number of 9286 CVS samples, 185 patients exhibited abnormal results (including atypical squamous cells of undetermined significance (ASC-US), atypical squamous cells, cannot-exclude high-grade lesions (ASC-H), low-grade squamous intraepithelial lesions (LGSIL), high-grade squamous intraepithelial lesions (HGSIL) and atypical glandular cells of undetermined significance (AGUS)). For each patient, we recorded complete blood count test. As control group, 303 age-matched patients with normal smears (negative for malignancy) whose complete blood count results were available were randomly selected. Patients who were diagnosed with an acute infectious disease, pregnant or known to have any hematological disease were excluded. Follow-up of the cases at our institution were performed according to the American Society for Colposcopy and Cervical Pathology (ASCCP) guidelines updated in $2012 .{ }^{16}$ We also recorded a histopathological evaluation of the cervical biopsies.

The NLR is defined as the rate of absolute neutrophil count to absolute lymphocyte count. The PLR is defined as the rate of absolute platelet count to the absolute lymphocyte count. Neutrophilia was defined as the number of neutrophils $\geq 70 \%$ of the total leukocytes and lymphopenia was defined as a lymphocyte count $\leq 15 \%$ of the total leukocytes.

We performed statistical analysis using SPSS statistical software, version 22.0 (SPSS Inc., Chicago, IL, USA). We expressed continous variables as mean \pm standard deviation, and we used percentages and frequencies for categorical variables. We analyzed categorical variables using a chi-squared test. We evaluated the difference between two groups for continuous variables using the Student's t-test. A p-value less than 0.05 was considered to be statistically significant for all of the statistical analyses.

\section{Results}

The mean ages of the patients in the study and control groups were similar $(42.35 \pm 13.06$ vs $40.92 \pm 13.39$ years, respectively; $\mathrm{p}=0.164)$. Abnormal CVS $(\mathrm{n}=185)$ consisted of 137 (72\%) ASC-US, 16 (8\%) ASC-H, 19 (10\%) L-SIL, 4 (2\%) H-SIL and 9 (4\%) AGUS. The neutrophil, lymphocyte counts, NLR, PLR, and rates of neutrophilia and lymphopenia are summarized in Table 1. The mean NLR and PLR of the study group were significantly higher than those of the controls $(p<0.001)$. Moreover, the NLRs and PLRs of the patients with ASC-US were significantly higher than those of the control group ( $\mathrm{p}<0.001$, and $\mathrm{p}<0.001$, respectively). However, patients with a diagnosis of ASC-US $(n=137)$ had similar neutrophil, lymphocyte counts, NLRs and PLRs compared with the patients with other abnormal CVS results $(p=0.187$, $\mathrm{p}=0.680, \mathrm{p}=0.146$, and $\mathrm{p}=0.170$, respectively).

Table 1: Neutrophil, lymphocyte counts, NLR, PLR, neutrophilia and lymphopenia rates of abnormal cytology and study groups

\begin{tabular}{|c|c|c|c|c|c|}
\hline & $\begin{array}{l}\text { ASCUS } \\
\text { Group } \\
(n=137)\end{array}$ & $\begin{array}{l}\text { Abnormal cytology } \\
\text { other than ASCUS } \\
\text { Group }(n=48)\end{array}$ & $\begin{array}{l}\text { Abnormal Cytology } \\
\text { Total Group } \\
(n=185)\end{array}$ & $\begin{array}{l}\text { Normal Cytology } \\
\text { Group } \\
(n=303)\end{array}$ & $p^{(a, b, c)}$ \\
\hline $\begin{array}{l}\text { Age (years) } \\
\text { (mean } \pm \text { sd) }\end{array}$ & $41.84 \pm 11.31$ & $42.00 \pm 17.44$ & $42.35 \pm 13.06$ & $40.92 \pm 13.39$ & $0.164^{a}, 0.425^{b}$ \\
\hline $\begin{array}{l}\text { Neutrophil Count } \\
\text { (Mean } \pm \text { sd) }\end{array}$ & $4507.09 \pm 2057.65$ & $5013.19 \pm 2261.09$ & $4623.93 \pm 2088.76$ & $4676.94 \pm 749.75$ & $0.394^{a}, 0.187^{b}, 0.165^{c}$ \\
\hline $\begin{array}{l}\text { Lympocyte Count } \\
\text { (Mean } \pm \text { sd) }\end{array}$ & $1320.78 \pm 326.69$ & $1402.80 \pm 766.99$ & $1341.78 \pm 457.64$ & $2244.58 \pm 749.75$ & $<0.001^{* a}, 0.680^{b},<0.001^{*_{c}}$ \\
\hline $\begin{array}{l}\text { NLR } \\
(\text { Mean } \pm \text { sd })\end{array}$ & $3.47 \pm 1.74$ & $3.90 \pm 2.32$ & $3.55 \pm 1.85$ & $2.40 \pm 1.75$ & $<0.001^{*_{a}}, 0.146^{b},<0.001^{*_{c}}$ \\
\hline $\begin{array}{l}\text { PLR } \\
(\text { Mean } \pm \text { sd })\end{array}$ & $215.54 \pm 63.08$ & $198.34 \pm 56.79$ & $209.80 \pm 62.02$ & $136.66 \pm 82.31$ & $<0.001^{*_{a}}, 0.170^{\mathrm{b}},<0.001^{*_{\mathrm{c}}}$ \\
\hline $\begin{array}{l}\text { Neutrophilia } \\
n(\%)\end{array}$ & $15(10.9 \%)$ & $8(16 \%)$ & $23(12.4 \%)$ & $42(13.9 \%)$ & $0.652^{\mathrm{a}}$ \\
\hline $\begin{array}{l}\text { Lymphopenia } \\
n(\%)\end{array}$ & $4(3.0 \%)$ & $3(6.2 \%)$ & $7(3.8 \%)$ & $15(5.0 \%)$ & $0.547^{a}$ \\
\hline
\end{tabular}

NLR: Neutrophil-lymphocyte ratio, PLR: Platelet-lymphocyte ratio , ${ }^{*} p<0.01$, a: Total abnormal CVS vs control group, b: ASCUS vs other abnormal CVS group, c: ASCUS vs control group 
Forty-seven $(25.4 \%)$ of the patients with abnormal CVS received additional colposcopic biopsies at our center. The results revealed non-neoplastic lesions in 21 of the patients, cervical intraepithelial neoplasias (CIN) in 25 patients (CIN-I $\mathrm{n}=16$, CIN-II $\mathrm{n}=2, \mathrm{CIN}-\mathrm{III} \mathrm{n}=7$ ) and one squamous cell carcinoma. In terms of the cervical biopsy, the NLRs and PLRs of patients with non-neoplastic lesions were similar to those of patients with CIN (NLR: $3.21 \pm 1.01$ vs $4.40 \pm 3.84, \mathrm{p}=0.126$; PLR: $194.69 \pm 50.54$ vs $216.32 \pm 59.87, \mathrm{p}=0.369$, respectively).

\section{Discussion}

This study is the first to document the absence of neutrophilia and high NLRs in patients with abnormal cervical cytology compared with patients with normal cervical cytology. Moreover, no difference in the NLRs of patients with CIN and normal histopathological findings support previous studies, which have found that neutrophilia is a parameter of advanced stage cervical cancer. ${ }^{1}$ In a study by Fernandes et al., significantly elevated neutrofil counts were observed in invasive cancer compared with CIN. ${ }^{1}$ Many previous studies have noted the importance of inflammatory cells in the growth and progression of some tumors. In the tumor microenvironment, immune system cells such as granulocytes and lymphocytes are regulators of angiogenesis and metastasis. ${ }^{5}$ The presence of a large number of neutrophils in the stroma of the tumoral tissue is associated with a poor prognosis. ${ }^{5}$ Additionally, leukocyte migration and function at the tumor site is controlled by specific cytokines released from the leukocytes. ${ }^{17}, 18$ For instance, vascular endothelial growth factors released from neutrophils, were found to be strongly associated with recurrence and metastasis of cervical cancer. ${ }^{3,19}$ As a result, neutrophilia seems to be a finding observed after the development of invasive cervical cancer.

Relative lymphopenia is another alteration in the circulating white blood cell count in systemic inflammatory response because, the host response is largely dependent on lymphocytes. Whether the decreased number of lymphocytes precipitates the conditions for atypical changes in the cervix is unclear. In cervical cancer and preinvasive lesions of the cervix, the local cellular immunity provided by $\mathrm{T}$ lymphocytes has been noted by many studies. ${ }^{1}$ In addition, circulating lymphocytes have been shown to secrete cytokines, which prevent proliferation and metastasis of tumor cells and provide important functions in cytotoxicity. Nevertheless, platelet counts also play a role in systemic inflammation. The PLR was found to be correlated with prognosis in cases with resected pancreatic cancer ${ }^{9}$ as well as associated with cervical stromal involvement in endometrial adenocarcinoma. ${ }^{4}$ Pro-inflammatory cytokines such as IL-1 and IL-6 contribute to the megakaryocyte proliferation. ${ }^{20}$ In our study, the lymphopenia and significantly higher PLR in abnormal cytology group support the idea that host systemic inflammation is associated with both lymphopenia and thrombocytosis. ${ }^{21}$
Cervical inflammation due to infectious or non-infectious causes presents nonspecific tissue responses. Cytological changes in inflammation are similar irrespective of the etiology; these changes manifest themselves as abnormal Pap smear results. The interpretation of abnormal CVS is challenging because hypoestrogenic states, infections, atrophy, pregnancy, dysplasia of the cervix, and contraceptive use all yield similar diagnostic symptoms..$^{22}$ The persistence of atypical squamous cells after treatment of these conditions necessitates further investigation. In this study, irrespective of the cause, patients with abnormal cytologies (mostly ASC-US) also exhibited high NLRs and PLRs, which are indicative of systemic inflammation. The systemic inflammation detected by NLRs and PLRs can-not discriminate between whether the cause is infectious or non-infectious. However, the imbalance between host inflammatory and immune response may lead to a negative association with oncological outcome.

Limitations of this study included its retrospective design and small sample size. However, this study revealed for the first time that high NLRs are associated with abnormal cervical cytology results. Due to the insufficient amount of histopathological evidence, it is not possible to conclude whether a correlation between high-grade cervical lesions and NLR exists. Predictive values of NLR for preinvasive/invasive neoplasia must be clarified with future research.

\section{References}

1. Fernandes PC, Garcia CB, Micheli DC, Cunha FQ, Murta EFC. Circulating neutrophils may play a role in the host response in cervical cancer. Int J Gynecol Cancer 2007; 17:1068-74.

2. Kose M, Celik F, Kose SK, Arioz DT, Yilmazer M. Could the platelet-to-lymphocyte ratio be a novel marker for predicting invasiveness of cervical pathologies? Asian Pac J Cancer Prev 2015;16(3):923-6.

3. Tavares-Murta BM, Mendonca MA, Duarte NL, et al. Systemic leukocyte alterations are associated with invasive uterine cervical cancer. Int J Gynecol Cancer 2010; 20:1154-9.

4. Wang D, Yang JX, Cao DY, et al. Preoperative neutrophillymphocyte and platelet-lymphocyte ratios as independent predictors of cervical stromal involvement in surgically treated endometriod adenocarcinoma. Onco Targets and Therapy 2013;6:211-6.

5. Gunaldi M, Goksu S, Erdem D, et al. Prognostic impact of platelet/lymphocyte and neutrophil/lymphocyte ratios in patients with gastric cancer: a multicenter study. Int J Clin Exp Med 2015;8(4):5937-42.

6. Kwon HC, Kim SH, Oh SY, et al. Clinical significance of preoperative neutrophil-lymphocyte versus platelet-lymphocyte ratio in patients with operable colorectal cancer. Biomarkers 2012;17(3):216-22. 
7. Walsh SR, Cook EJ, Goulder F, Justin TA, Keeling NJ. Neutrophil-lymphocyte ratio as a prognostic factor in colorectal cancer. J Surg Oncol 2005;91(3):181-4.

8. Halazun KJ, Hardy MA, Rana AA et al. Negative impact of neutrophil-lymphocyte ratio on outcome after liver transplantation for hepatocellular carcinoma. Ann Surg 2009;250(1):141-51.

9. Bhatti I, Peacock O, Lloyd G, Larvin M, Hall RI. Preoperative hematologic markers as independant predictors of prognosis in resected pancreatic ductal adenocarcinoma: neutrophil-lymphocyte versus platelet-lymphocyte ratio. Am J Surg 2010;200(2):197-203.

10. Sarraf KM, Belcher E, Raevsky E, Nicholson AG, Goldstraw P, Lim E. Neutrophil/lymphocyte ratio and its association with survival after complete resection in nonsmall cell lung cancer. J Thorac Cardiovasc Surg 2009; $137(2): 425-8$

11. Wei Y, Jiang YZ, Qian WH. Prognostic role of NLR in urinary cancers: a meta-analysis. PloS One 2014;9(3): e92079.doi:10.1371/journal.pone.0092079

12. Cho HB, Hur HW, Kim SW, et al. Pre-treatment neutrophil to lymphocyte ratio is elevated in epithelial ovarian cancer and predicts survival after treatment. Cancer Immunol Immunother 2009;58(1):15-23.

13. Lee YY, Choi CH, Kim HJ, et al. Pretreatment Neutrophil: lymphocyte ratio as a prognostic factor in cervical carcinoma. Anticancer Res 2012;32(4):1555-62.

14. Zhang Y, Wang L, Liu Y, et al. Preoperative neutrophillymphocyte ratio before platelet/lymphocyte ratio predicts clinical outcome in patients with cervical cancer treated with initial radical surgery. Int J Gynecol Cancer 2014; 24(7):1319-25.
15. Mizunuma M, Yokoyama Y, Futagami M, Aoki M, Takai Y, Mizunuma H. The pretreatment neutrophil-to-lymphocyte ratio predicts therapeutic response to radiation therapy and concurrent chemoradiation therapy in uterine cervical cancer. Int J Clin Oncol 2015; 20(5):989-96.

16. Massad LS, Einstein MH, Huh WK, et al. 2012 Updated consensus guidelines for the management of abnormal cervical cancer screening tests and cancer precursors. J Low Genit Tract Dis 2013;17:1-27.

17. Coussens LM, Werb Z. Inflammation and cancer. Nature 2002;420:860-7.

18. Balkwill F, Mantovoni A. Inflammation and cancer: back to Virchow? Lancet 2001;357:539-45.

19. Kusumanto YH, Dam WA, Hospers GA, Meijer C, Mulder NH. Platelets and granulocytes, in particular the neutrophils, form important compartments for circulating vascular endothelial growth factor. Angiogenesis 2003; 6(4):283-7.

20. Klinger MH, Jelkmann W. Role of blood platelets in infection and inflammation. J Interferon Cytokine Res 2002; 22:913-22.

21. Smith RA, Bosonnet L, Ghaneh P, et al. The platelet-lymphocyte ratio improves the predictive value of serum $\mathrm{Ca}$ 19-9 levels in determining patient selection for staging laparoscopy in suspected periampullary cancer. Surgery 2008;143:658-66.

22. Patton AL, Duncan L, Bloom L, Phaneuf G, Zafar N. Atypical squamous cells, cannot exclude a high-grade intraepithelial lesion and its clinical significance in postmenopausal, pregnant, postpartum, and contraceptive-use patients. 